\title{
Opportunities for Chromatic Aberration Corrected High-Resolution Transmission Electron Microscopy, Lorentz Microscopy and Electron Holography of Magnetic Minerals
}

\author{
R. E. Dunin-Borkowski ${ }^{1}$, T. Kasama ${ }^{2}$, R. J. Harrison ${ }^{3}$, L. Houben ${ }^{1}$, J. Barthel ${ }^{1}$, A. Thust ${ }^{1}$, \\ M. Luysberg ${ }^{1}$, C. B. Boothroyd ${ }^{1}$, A. Kovács ${ }^{1}$, J. R. Jinschek $^{4}$ \\ ${ }^{1}$ Ernst Ruska-Centre for Microscopy and Spectroscopy with Electrons, Peter Grünberg Institute, \\ Forschungszentrum Jülich, D-52425 Jülich, Germany \\ ${ }^{2}$ Center for Electron Nanoscopy, Technical University of Denmark, DK-2800 Lyngby, Denmark \\ ${ }^{3}$ Department of Earth Sciences, Cambridge University, Downing Street, Cambridge CB2 3EQ, U.K. \\ ${ }^{4}$ FEI Company, Achtseweg Noord 5, 5651 GG Eindhoven, The Netherlands
}

High spatial resolution structural information about magnetic minerals can be obtained using spherical aberration $\left(\mathrm{C}_{\mathrm{S}}\right)$ corrected transmission electron microscopy (TEM), while local magnetic information can be obtained using complementary TEM techniques such as Lorentz microscopy and off-axis electron holography [1]. Figure 1 shows Lorentz TEM (out-of-focus) images of a magnetite $\left(\mathrm{Fe}_{3} \mathrm{O}_{4}\right)$ specimen acquired at $300 \mathrm{kV}$ without the use of $\mathrm{C}_{\mathrm{S}}$ correction. The images were recorded below the Verwey transition, at which the magnetocrystalline anisotropy increases by an order of magnitude and the magnetic easy axis switches from the $<111>$ directions of the parent cubic phase to the [001] direction of the low temperature monoclinic phase. In extended crystals examined at low temperature, $\mu \mathrm{m}$-scale monoclinic twin domains that are separated from each other by jagged twin walls are observed. The domains are subdivided by lamellar twins, which join at their ends to form needle twins that move in response to thermal or magnetoelastic stress by the advancement and retraction of the needle tips. Figures 1 (c) to (f) show a $180^{\circ}$ magnetic domain wall being driven to the right by an applied magnetic field, resulting in the conversion of a conventional $90^{\circ}$ domain wall into a 'divergent' $90^{\circ}$ domain wall. In individual nanocrystals that can only a single magnetic domain, we observe competing effects on the magnetic microstructure of shape anisotropy and magnetocrystalline anisotropy that are different above and below the Verwey transition [2].

In the most recent generation of transmission electron microscopes, chromatic aberration $\left(\mathrm{C}_{\mathrm{C}}\right)$ correction promises to provide improved spatial resolution and interpretability when compared with the use of $\mathrm{C}_{\mathrm{S}}$ correction alone, primarily as a result of improvements to the temporal damping envelope of the objective lens, especially at lower accelerating voltages [3-4]. The reduced dependence of image resolution on energy spread in a $\mathrm{C}_{\mathrm{C}}$ corrected microscope offers benefits for conventional bright-field and dark-field imaging as a result of the decreased influence of inelastic scattering on spatial resolution, even when using zero-loss energy filtering. Less refocusing is also necessary when moving between regions of different specimen thickness, which may be advantageous for electron tomography of thick specimens. For energy-filtered TEM, $\mathrm{C}_{\mathrm{C}}$ correction allows large energy windows and large objective aperture sizes to be used without compromising the spatial resolution of energy-loss images. A further benefit of $\mathrm{C}_{\mathrm{C}}$ correction results from the fact that combined $\mathrm{C}_{\mathrm{S}}$ and $\mathrm{C}_{\mathrm{C}}$ correction of the Lorentz lens of a TEM allows images to be recorded in magnetic-field-free conditions with a spatial resolution of better than $0.5 \mathrm{~nm}$ with the conventional TEM objective lens switched off. Figure 2 shows Fourier transforms of $\mathrm{C}_{\mathrm{C}}$ and $\mathrm{C}_{\mathrm{S}}$ corrected lattice images taken in both high-resolution and Lorentz (magnetic-field-free) operating modes [5]. 


\section{References:}

[1] T. Kasama et al., Earth and Planetary Science Letters 297 (2010) 10.

[2] R. J. Harrison, Reviews in Mineralogy and Geochemistry 39 (2000) 175.

[3] L. Reimer and P. Gentsch, Ultramicroscopy 1 (1975) 1.

[4] B. Kabius et al., J. Electron Microsc. 58 (2009) 147.

[5] We acknowledge J. M. Feinberg, N. S. Church, E.T. Simpson and M. Beleggia for contributions.

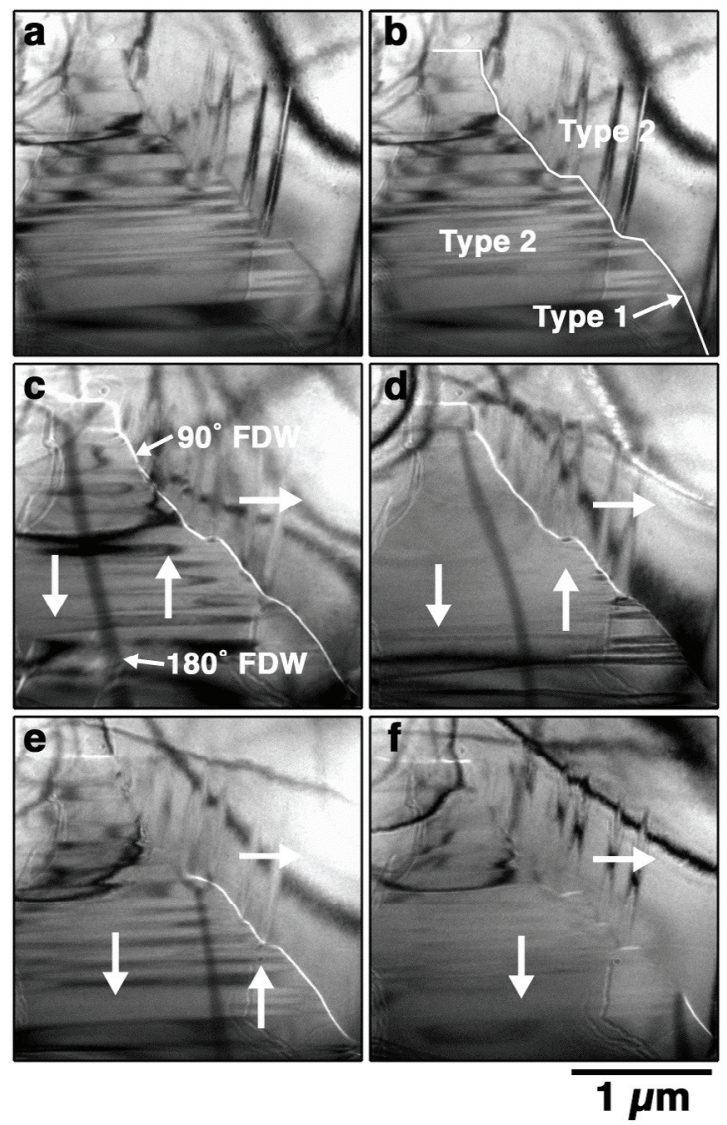

Fig. 1. (a, b) Bright-field TEM images of a magnetite specimen that contains 'Type 1' and 'Type 2' ferroelastic twins. The images were recorded close to focus with the sample cooled below the Verwey transition. Diffraction contrast in differently oriented twins and curving bend contours are visible. A Type 1 twin boundary is marked with a white line in (b). The region to the left of this boundary has its [001] magnetic easy axis oriented top-to-bottom. The region to the right has its easy axis oriented left-to-right. (c-f) Lorentz TEM images recorded from the same region in different (downward) fields showing ferrimagnetic domain walls (FDWs). The magnetic fields were applied by tilting the specimen in a 920 Oe vertical field generated by exciting the TEM objective lens. The in-plane components of the applied field are: (c) 20, (d) 50, (e) 80 and (f) 150 Oe.
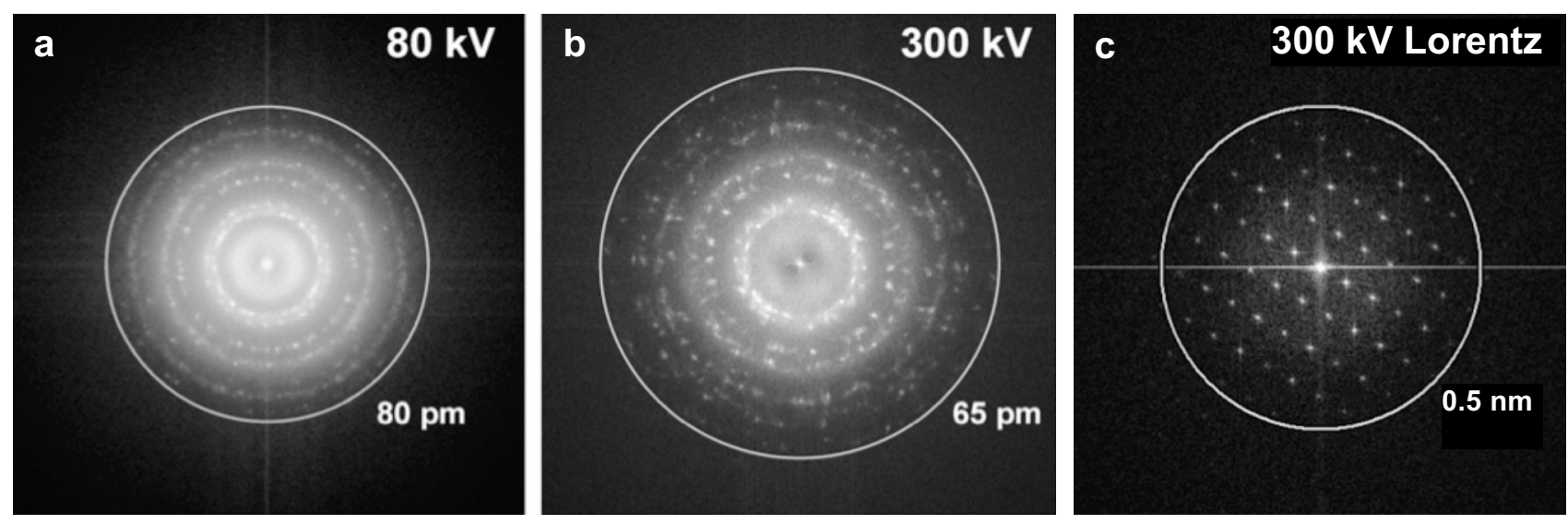

Fig. 2. Fourier transforms of $\mathrm{C}_{\mathrm{C}}$ and $\mathrm{C}_{\mathrm{S}}$ corrected images of $(\mathrm{a}, \mathrm{b}) \mathrm{Au}$ on $\mathrm{C}$ and $(\mathrm{c}) \mathrm{Cs}_{0.5}\left[\mathrm{Nb}_{2.5} \mathrm{~W}_{2.5} \mathrm{O}_{14}\right]$. In the normal operating mode of the TEM, the smallest image detail is (a) $\sim 80 \mathrm{pm}$ at $80 \mathrm{kV}$ and (b) $\sim 65 \mathrm{pm}$ at $300 \mathrm{kV}$. In Lorentz mode, with the objective lens off, $0.5 \mathrm{~nm}$ detail is present at $300 \mathrm{kV}$. 